\title{
Perturbation of old knowledge precedes integration of new knowledge
}

\author{
Xiaoping Fang ${ }^{\mathrm{a}, \mathrm{b}, *}$, Charles A. Perfetti ${ }^{\mathrm{a}, \mathrm{b}, *}$ \\ ${ }^{a}$ Learning Research and Development Center, University of Pittsburgh, Pittsburgh, PA, USA \\ ${ }^{\mathrm{b}}$ Center for Neural Basis of Cognition, Pittsburgh, PA, USA
}

A R T I C L E I N F O

\section{Keywords:}

Word learning

Word knowledge

Integration

Consolidation

ERPs

Semantic priming

\begin{abstract}
A B S T R A C T
The importance of memory consolidation in integrating new knowledge has received much recent attention in the field of word learning. Less examined is the change in existing word knowledge as a result of learning, which we hypothesize to occur prior to the opportunity for consolidation. To test this, we had participants learn new meanings for known words and novel words. Then they performed a one-back task on a list of words that included the trained words followed by words that probed either their new or original meanings while EEGs were recorded. A probe word related to the new meaning of the preceding trained word did not show an N400 reduction compared to an unrelated word, suggesting that the new meaning had not been fully integrated, consistent with one account of complementary learning systems. However, when the probe word was related to the original meaning of the preceding trained word a perturbation effect was observed, indicated by a larger negativity at the central midline cluster $(\mathrm{Cz})$ within 500-700 ms when the trained word had a new meaning than when presented as an exposure control. The perturbation effect suggests that even before a new meaning has become integrated with a word form, the attempt to learn a new meaning temporarily makes the original meaning of a word less accessible.
\end{abstract}

\section{Introduction}

The outcome of learning - the acquisition of knowledge-is observable when the acquired knowledge is explicitly retrieved from memory. However, such retrieval can be achieved through recollection of learning episodes even when new information has not yet been integrated into existing knowledge. Indeed, in the word learning field, when and how a word is integrated into an existing lexical network and thus becomes functional is a critical question (Davis and Gaskell, 2009). It remains even less clear how existing word knowledge can be affected by learning experience, a process that is part of the dynamic updating of a lexical network.

According to the account of complementary learning systems (CLS), only after episodic memories of learning experiences are transferred to semantic memory, can a novel word be integrated into the lexical network and behave as other existing words do (Davis and Gaskell, 2009; Mcclelland et al., 1995). As part of a lexical network, meaning is activated automatically with a word (e.g., "plenty") and can prime semantically related words (e.g., "enough") through spreading activation (McNamara, 2005). Taking advantage of this idea, recent studies used the emergence of semantic priming between novel words and existing words as an indicator of integration (e.g., Coutanche and Thompson-Schill, 2014; Tamminen and Gaskell, 2013). Tamminen and
Gaskell (2013) used a primed lexical decision task and found that newly learned words can prime semantically related words one week after the learning session, but not on the day of learning. This finding is consistent with the assumption that memory consolidation is essential in integrating new words into a lexical network (Davis and Gaskell, 2009), although other studies suggest immediate integration is possible under some learning conditions (e.g., Coutanche and Thompson-Schill, 2014; Geukes et al., 2015; Lindsay and Gaskell, 2013).

The research on learning and consolidation has used novel words to represent word learning. Learning new meanings for already-known words, however, has been rarely studied (Fang et al., 2016; Rodd et al., 2012), despite the fact that this situation-learning new meanings for words with meanings already established-is quite common in enriching a person's vocabulary. For example, one may learn that "skate" is a kind of fish long after knowing its more common meaning. Whereas learning novel words requires establishing new representations for both the form of a word and its meaning, learning new meanings for known words requires attaching new meanings to existing word forms. For existing words, the activation of original meanings during the encoding of new meanings produces an interaction between new and existing word knowledge, a process that may facilitate the integration of new information (Schlichting et al., 2015; Schlichting and Preston, 2015; van Kesteren et al., 2012).

\footnotetext{
* Corresponding authors at: Learning Research and Development Center, University of Pittsburgh, 3939 O'Hara Street, Pittsburgh, PA 15260, USA.

E-mail addresses: xpfang4@gmail.com (X. Fang), perfetti@pitt.edu (C.A. Perfetti).
} 
In addition to the integration of new knowledge, learning also brings about updating of existing knowledge. The old-form, newmeaning paradigm allows us to examine how the existing lexical network is affected by learning. Research on retroactive interference has shown that the encoded memories of $\mathrm{AB}$ associations become vulnerable to interference when they are reactivated during the encoding of AC associations (e.g., Forcato et al., 2007; Koen and Rugg, 2016). Given the automatic activation of existing word knowledge in the learning phase, we proposed that associating with new unrelated meanings might cause a perturbation of the existing connections between known words and their original meanings. In contrast to episodic nature of $\mathrm{AB} / \mathrm{AC}$ associations such as pairs of syllables or novel objects in the previous studies (e.g., Forcato et al., 2007; Schlichting et al., 2015), known words have been acquired and represented in semantic memory long before the learning of new meanings. Therefore, it is not obvious that such well-established word knowledge is affected by recent learning experience.

ERPs can provide indicators of integration and updating of word knowledge. In particular, the N400, a negative going ERP component typically observed between 300 and $500 \mathrm{~ms}$ after the onset of a word presentation at central and parietal sites reflects meaning access from long-term semantic memory (Kutas and Federmeier, 2011). The N400 reduction can be evoked by semantically related words: a smaller negativity on a word proceeded by semantically related words than by unrelated words. The $\mathrm{N} 400$ is sometimes followed by late positive complex (LPC), a positive going component in a later time window typically including $500-700 \mathrm{~ms}$. In contrast to the $\mathrm{N} 400$, which is interpreted as reflecting automatic meaning processes, the LPC may reflect controlled lexico-semantic processing such as explicit meaning retrieval (Bakker et al., 2015; Hill et al., 2005, 2002; Zachau et al., 2014). Previous studies on novel word learning have shown N400 and/ or LPC semantic priming between novel words and existing words on the day of learning in semantic relatedness judgment task (Bakker et al., 2015; Mestres-Misse et al., 2007; Perfetti et al., 2005). However, it remains unclear whether these semantic priming effects rely on explicit retrieval of novel word meanings required to perform the task and whether they would be observable when meaning retrieval is minimized in a task.

In the experiment described below, participants learned new meanings for known words and novel words. To account for the effects of mere exposure, we included exposure controls - known words and novel words that were presented to participants but without new meanings. Following the learning phase, they performed a one-back task on a list of words presented one-by-one while EEGs were recorded. Embedded in the word list were word pairs, each consisting of a trained word followed by a probe that was either related to its original meaning or unrelated. ERPs on both trained words and probes were considered. We predicted that immediately following learning, new-meaning probes for novel words would not be primed by novel words and thus would not show reduced negativity within N400 or LPC time windows; new-meaning probes for known words may be primed if the new meanings had been integrated prior to overnight consolidation. We were specifically interested in a perturbation effect: a reduction in the ability of a trained word to prime a probe word related to its original meaning, as indicated by an N400 and/or LPC on the probe word, compared with exposure controls. For ERPs on trained words, we predicted that novel words with new meanings would be comparable to their exposure controls in the N400, because new meanings would not be activated automatically on the day of learning. In contrast, known words with new meanings may show a larger negativity within N400 and even LPC time windows compared with exposure controls indicating reduced accessibility of original meanings if perturbation occurred (Rugg, 1990).

\section{Materials and methods}

\subsection{Participants}

Twenty-one (15 females) right-handed native English speakers participated in the study. All had normal or corrected-to-normal vision and none had been diagnosed with any learning disability. Participants' ages ranged from 19 to 23 years old, with a mean of 20.10. Data from seven additional participants were excluded from analysis because of less than $40 \%$ accuracy of the multiple-choice test immediately after learning $(n=2)$, fewer than 15 valid trials in any of the conditions in the one-back task $(n=4)$, or technical failure in recording event information in ERP data $(n=1)$. Participants provided written informed consent before the experiment and received either course credit or monetary compensation for their time. All experimental procedures were carried out with the approval of the University of Pittsburgh institutional review board.

\subsection{Stimuli}

\subsubsection{Trained words and new meanings for learning and testing}

Trained words included 48 known words and 48 novel words, each grouped into two sets of 24 words: one set was paired with new meanings and the other set was used as exposure controls, with the assignment of words to these two sets counterbalanced across participants (See Table 1 for examples of stimuli). Known words had a mean word frequency of 40.17 per million (Brysbaert and New, 2009), and a mean concreteness of 3.04 along a scale of 1 (abstract) to 5 (concrete) (Brysbaert et al., 2013). According to Wordsmyth English Dictionary Thesaurus (Parks et al., 1998), each known word had only one meaning, while sometimes having more than one related sense (mean $=2.54$, range $=1-5$ ). Novel words are pronounceable pseudowords that were not derived from any existing words used in any other parts of the study. Known words and novel words were matched on bigram frequency, number of syllables and word length (all $p s>.20$ ). Forty-eight new adjective meanings, taken from a previous study (Fang et al., 2016), were grouped into two sets (24 meanings in each): one set was paired with known words and the other with novel words, with the assignment counterbalanced across participants. New meanings were not related to the original meanings of the known words, as reviewed by three native speakers independently and further confirmed by Latent Semantic Analysis (LSA, Landauer and Dumais, 1997). LSA measures semantic similarity of two semantic spaces constructed from a large corpus based on each pair of words and new meanings. A mean LSA cosine value of .008 ( $\mathrm{SD}=.049, p=.142$ ) indicated a low semantic relatedness between words and their new meanings.

Table 1

Examples of trained words, new meanings and probes.

\begin{tabular}{|c|c|c|c|c|c|}
\hline Condition & Trained words & New meaning & Related probe (original) & Related probe (new) & Unrelated probe \\
\hline Known word/meaning & Plenty & Causing fever & Enough & Germ & - \\
\hline Known word/control & Weapon & $* * * * * * * * * * * * * * * * *$ & War & - & Door \\
\hline Novel word/meaning & Tasdite & Having a tight schedule & - & Busy & Paper \\
\hline Novel word/control & Attave & $* * * * * * * * * * * * * * * * * *$ & - & - & Desk \\
\hline
\end{tabular}




\subsubsection{Meaning probes for one-back task}

Forty-eight words, each related to one of the 48 new meanings, served as related probes of new meanings. For each participant, half were related to new meanings of known words (e.g., plenty - germ, see Table 1) and the other half related to new meanings of novel words (e.g., tasdite - busy). Another 48 words were related to the original meanings of the 48 known words, i.e., related probes of original meanings. Half were paired with known words with new meanings (e.g., plenty - enough), and the other half with exposure controls (e.g., weapon - war). To assess whether we had successfully identified meaning relations, 19 native English speakers were presented with word pairs consisting of known words and original-meaning probes and pairs of new meanings and their probes, and asked to rate the semantic relatedness of each pair along a scale of 1 (unrelated) to 6 (related). The results were that meaning probes were related to the new meanings to an extent similar to those related to original meanings of known words $(5.01 \pm .43$ for new-meaning probes and $5.13 \pm .46$ for original-meaning probes, $p=.192$ ). In addition to related probes, there were 24 unrelated probes paired with exposure controls of known words (e.g., weapon - door) and another 24 unrelated probes paired with novel words with new meanings (e.g., tasdite - paper). Exposure controls of novel words were also presented twice and followed by existing words (e.g., attave - desk), however, the processing of these existing words was of no interest, because the preceding novel words did not have meanings. As a result, there were 192 word pairs. The new-meaning probes, original-meaning probes, and unrelated probes were matched at word frequency, bigram frequency, word length, and orthographic neighborhood size, and concreteness (all $F \mathrm{~s}<1.5$, all $p s>.23$ ).

\subsection{Procedure}

\subsubsection{Learning phase}

In the learning phase, participants learned new meanings for known words and novel words through a self-paced associative learning paradigm. They were told that on some trials they would see a known word or a novel word presented with a string of stars rather than with a new meaning (i.e., exposure controls). There were six learning blocks and each of the 96 trained words was presented once in each block, leading to six learning trials for each word in total. In the first block, each learning trial started with a central fixation $(500 \mathrm{~ms})$, followed by a trained word $(1500 \mathrm{~ms})$ and then by its meaning or a string of stars, which remained until the participant pressed the space bar to advance to next page. Thus, participants decided how long to study a word's meaning before moving on to the next word. From the second to sixth blocks, when a word as presented $(1000 \mathrm{~ms})$, participants were additionally instructed to recall the new meaning (without an overt response). After attempting meaning recall, they pressed the space bar to reveal the correct answer. At this point, they evaluated whether what they had recalled was correct by pressing " $\mathrm{F}(\mathrm{No})$ " or "J (Yes)". After their evaluation, the correct meaning remained on the screen until participants were ready to continue to the next word. This selfevaluation task was to facilitate the learning. EEGs were recorded during learning but the results are not reported here.

\subsubsection{Tests on new meanings}

How well participants learned the new meanings was tested with both meaning generation and multiple-choice tests at two time points: immediately following the learning phase and one week later. In the meaning generation tests, participants typed the meaning of each trained word presented on the screen. For trained words without new meanings (i.e., exposure controls), they simply typed "n" for none. Participants' responses were independently rated by two experimenters who were blind to the conditions, based on how close the responses were to the correct meanings on a scale of 0 (no response or unrelated response) to 5 (the exact meaning). Inconsistencies between the raters were resolved through discussion before final scores were assigned. In the multiple-choice tests, participants were asked to choose the correct meaning for each word from four options. The fourth option was always a string of stars so that they could indicate their judgment if a word had not been paired with a meaning (i.e., exposure controls). Only accuracy was calculated in the multiple-choice tests. Although exposure controls were tested in both tasks, only responses to trained words with new meanings were of interest and reported.

\subsubsection{One-back task}

On the day of learning, participants performed the one-back task following the tests on new meanings. In the task, each participant saw a word list consisting of 192 pairs of trained words and probes, with one word presented at a time, while EEGs were recorded. The presentation sequence for words was as follows: a fixation cross (575-625 ms), followed by a word $(800 \mathrm{~ms})$, then a blank screen $(600 \mathrm{~ms})$. Participants were instructed to press the space bar when they saw a word presented twice in sequence. Otherwise, they were told not to respond. Fortyeight out of the 192 probes were repeated and therefore required behavioral responses. Participants were instructed to minimize blinking when a word or fixation was presented. There were four blocks, with a break between blocks. Each block included 108 words and lasted approximately $4 \mathrm{~min}$. All participants completed a practice session to become familiarized with the task prior to the formal session. Because of a technical failure, participants' behavioral responses were not recorded. (See Fig. 1 for a diagram of procedure).

\subsection{ERP data acquisition and preprocessing}

Participants were fitted with a Geodesic Sensor Net with a $128 \mathrm{Ag}$ / $\mathrm{AgCl}$ electrode array and data were recorded using the associated NetStation acquisition software (Electrical Geodesics, Inc., Eugene, OR) with a sampling rate of $1 \mathrm{kHZ}$ and a hardware bandpass filter of .01-200 Hz. Data were preprocessed with NetStation Tool software. A bandpass filter of .01-30HZ was applied, and then data were segmented into 850-ms epochs, starting $100 \mathrm{~ms}$ before word presentation. Epochs with artifacts were rejected for further analysis, including eye blink (exceeding $\pm 140 \mu \mathrm{V}$ ), eye-movement (exceeding $55 \mu \mathrm{V}$ ), and extreme variance (larger than $200 \mu \mathrm{V}$ ). As a result, four participants with fewer than 15 valid epochs for any condition were excluded from further analysis. For participants whose data entered statistical analysis, on average there were 41.9 valid epochs $(\max =48)$ for trained words and 21.0 valid epochs $(\max =24)$ for meaning probes. Channels with artifacts on more than $20 \%$ of epochs were marked as bad channels, and data from surrounding channels were used for interpolation. For each participant, ERPs on each type of trained words and meaning probes were acquired through averaging epochs under the same

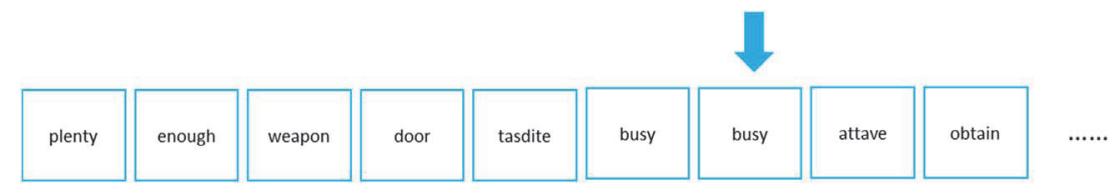

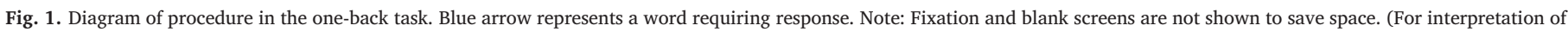
the references to color in this figure legend, the reader is referred to the web version of this article). 
condition. ERPs were referenced to the average of whole scalp before corrected relative to $100-\mathrm{ms}$ baseline before word presentation.

\subsection{ERP data analysis}

As N400 and LPC were the main focus, we examined specifically the mean amplitudes within $300-500 \mathrm{~ms}$ and $500-700 \mathrm{~ms}$ at the central midline cluster (i.e., $\mathrm{Cz}$ cluster), where meaning effects are robustly observed in word learning studies (Bakker et al., 2015; Batterink and Neville, 2011; Mestres-Misse et al., 2007). For each type of trained words and meaning probes, the mean amplitude within each time window for each participant was extracted using EP Toolkit (Dien, 2010). For trained words, contrasts between words with new meanings and exposure controls were tested for novel words and known words separately. Since the assignment of trained words to the two training conditions was counterbalanced across participants, the effects of meaning learning were examined through comparing the same set of words, which provided the most sensitive measurement. For probes, we first examined the semantic priming effect by comparing related and unrelated probes of exposure controls of known words. To examine whether existing connections had been perturbed, we compared two types of original-meaning probes: probes related to original meanings of known words with new meanings and those related to their exposure controls. Again, the perturbation effect was based on a comparison on the same set of words, because we counterbalanced the assignment of a known word to the two training conditions across participants. To test whether the new meanings were integrated, we compared new-meaning probes with unrelated probes, for novel words and known words separately. All the contrasts within each time window were tested with paired $t$-tests.

To examine the spatial distribution of N400 and LPC, we conducted separate ANOVAs for midline and lateral clusters as previous studies did (e.g., Cavanagh and Geisler, 2006; Chwilla and Kolk, 2003; Perfetti et al., 2005). For the lateral clusters (F3, F4, C3, C4, P3, and P4), we included condition, hemisphere (left/right) and anteriority (anterior/ central/posterior); for midline clusters ( $\mathrm{Fz}, \mathrm{Cz}$, and $\mathrm{Pz}$ ), we include condition and anteriority (see Fig. 2 for clusters and channels). The analyses were conducted for each contrast of interest. The GreenhouseGeisser correction was applied when the assumption of sphericity was

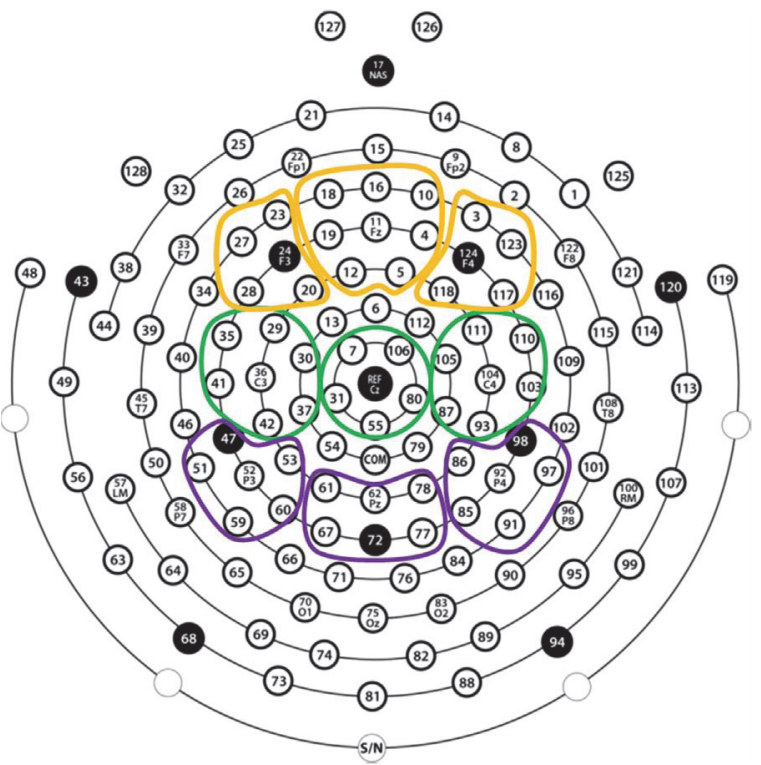

Fig. 2. Clusters and channels. From left to right, yellow circles indicate channels that contribute to F3, Fz, and F4 clusters; green circles indicate channels that contribute to $\mathrm{C} 3$, $\mathrm{Cz}$ and C4 clusters; purple circles indicate channels that contribute to P3, Pz, and P4 clusters. (For interpretation of the references to color in this figure legend, the reader is referred to the web version of this article).
Table 2

Performance in meaning generation (scores) and multiple-choice (accuracy) tests.

\begin{tabular}{llllll}
\hline & \multicolumn{2}{l}{ Meaning Generation (0-5) } & & \multicolumn{2}{l}{ Multiple-choice (accuracy) } \\
\cline { 2 - 3 } \cline { 5 - 6 } \cline { 5 - 6 } & Day1 & Day8 & & Day1 & Day8 \\
\hline Known words & $3.44(1.26)$ & $1.53(.95)$ & & $.92(.25)$ & $.73(.21)$ \\
Novel words & $2.98(1.49)$ & $1.04(.81)$ & & $.83(.29)$ & $.67(.17)$ \\
\hline
\end{tabular}

Note: Mean and SD (in parentheses) are reported. Meaning generation performance is scored on a scale of no or unrelated response (0) to exact meaning (5). Multiple-choice performance is proportion of correct choices.

violated (Mauchly's test, $p<.05$ ) and adjusted $\mathrm{p}$ values are reported.

\section{Results}

\subsection{Immediate and long-term retention of new meanings}

In both the meaning generation and multiple-choice tests, participants showed better learning for new meanings of known words than for novel words at both the immediate and the one-week test points (all $p s<.05$, see Table 2 for mean and SD). To better evaluate the maintenance of memories over one week, we calculated long-term retention ratio - the percentage of performance after one week relative to the performance on the immediate test $(100 \%$ means a perfect longterm retention). Participants retained memories about new meanings of known words (43.5\%) better than novel words (32.7\%) as tested in the meaning generation tasks $(p=.004)$, but not in the multiple-choice tasks (known words $=79.8 \%$, novel words $=80.3 \%, p=.870$ ).

\subsection{Meaning learning effects on trained words}

In the primary analyses, as shown in Fig. 3A and B, ERPs of novel words with new meanings were not significantly different from their exposure controls at $\mathrm{Cz}$ within the N400 or LPC time window ( $p s>$ .50). In contrast, known words with new meanings showed a larger negativity than exposure controls at both time windows (N400: $t_{(20)}=-2.138, p=.045$; LPC: $\left.t_{(20)}=-2.100, p=.049\right)$. Additionally, compared to exposure controls of known words, both novel words with new meanings $\left(t_{(20)}=-3.267, p=.004\right)$ and their exposure controls $\left(t_{(20)}=-2.691, \quad p=.014\right)$ showed a larger negativity within $300-500 \mathrm{~ms}$, but not in the later time window ( $p s>.16$ ).

In the spatial analyses, we found no significant main effects of training condition on known or novel words at the midline or lateral sites within either time window (all $p s>.43$ ); however, there were interactions of training condition with cluster locations. First, for novel words, lateral sites showed a significant three-way interaction of hemisphere, anteriority and training condition within both time windows (N400: $F(2,40)=4.766, p=.028, \eta_{p}{ }^{2}=.192$; LPC: $F(2,40)$ $=4.575, p=.026, \eta_{p}{ }^{2}=.186$; Fig. 3C). Pair $t$-tests showed a larger negativity for novel words with new meanings at the right frontal site (F4 cluster) within the LPC time window $\left(t_{(20)}=-2.902, p=.009\right)$; this difference was not observed at other sites (ps $>.13$ ). No differences within N400 time window reached statistical significance $(p s>.30)$. At both midline and lateral sites, novel words showed a marginally significant interaction between training condition and anteriority within the LPC time window (midline: $F(2,40)=2.831, p=.087, \eta_{p}{ }^{2}=.124$; lateral: $\left.F(2,40)=2.831, p=.089, \eta_{p}{ }^{2}=.127\right)$. For known words, the larger negativity for known words with new meanings tended to be more right lateralized within N400 time window, but the interaction of condition and hemisphere did not reach statistical significance $(F(1,20)$ $=3.621, p=.096, \eta_{p}{ }^{2}=.133$; Fig. 3D).

\subsection{Semantic priming effect}

To assess whether the one-back task produced semantic priming, we 


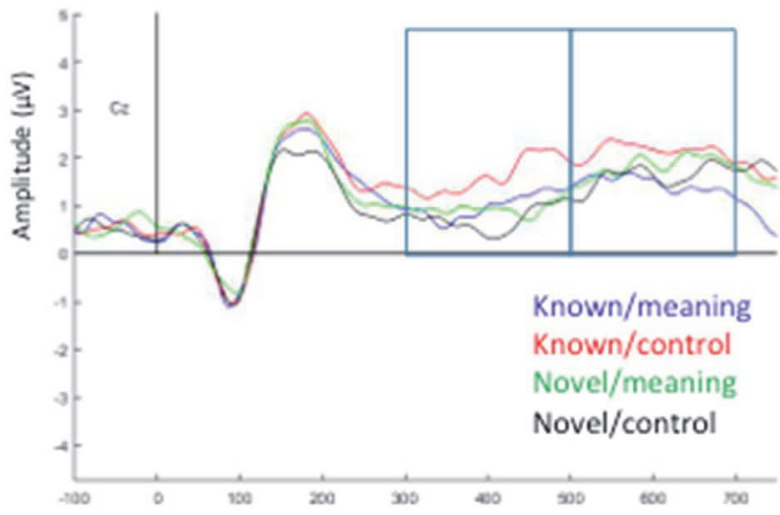

B Mean amplitudes $(\mu \mathrm{V})$ within $300-500$ and $500-700 \mathrm{~ms}$

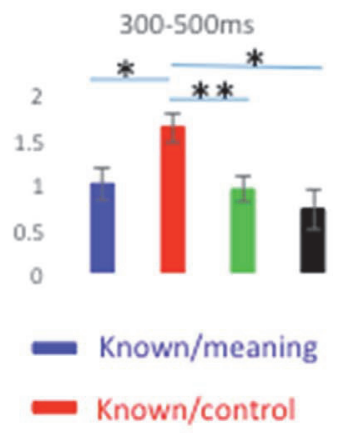

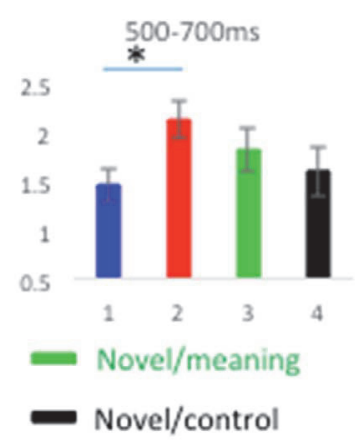

C

Novel words

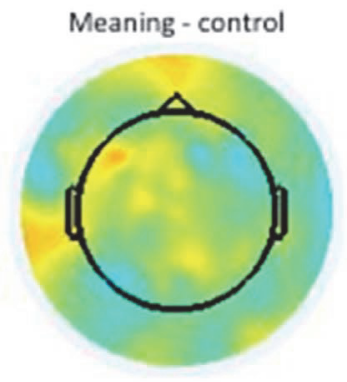

$300-500 \mathrm{~ms}$

D Known words

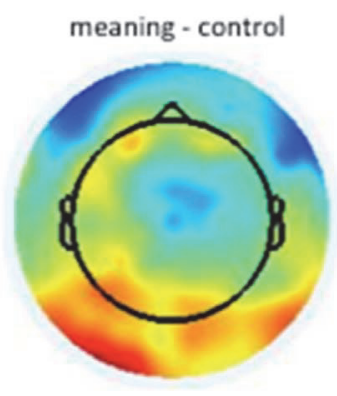

$300-500 \mathrm{~ms}$

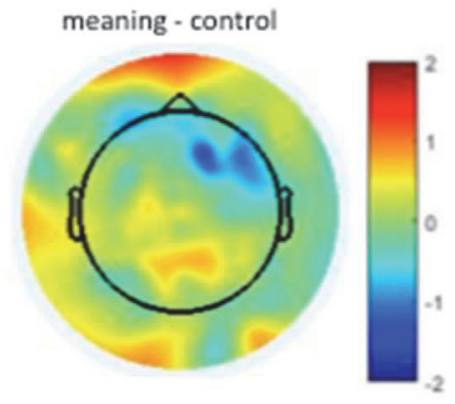

$500-700 \mathrm{~ms}$

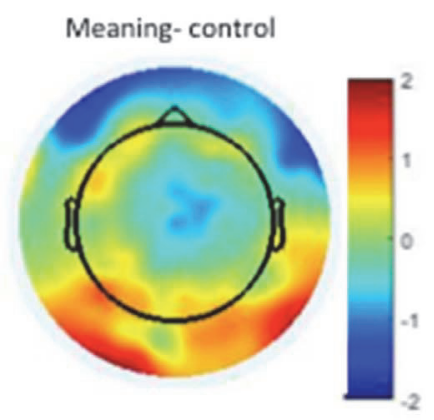

$500-700 \mathrm{~ms}$

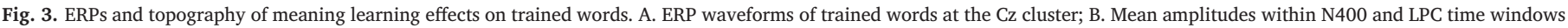

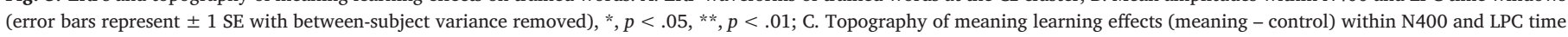
windows on novel words; D. Topography of meaning learning effects (meaning - control) within N400 and LPC time windows on known words.

compared related probes and unrelated probes that followed known words that were exposure controls (e.g., weapon - war vs. weapon door). As shown in Fig. 4A, unrelated probes evoked a marginally significant larger negativity at $\mathrm{Cz}$ compared to related probes within N400 time window $\left(t_{(20)}=-1.742, p=.097\right)$ with no effect within the LPC time window $\left(t_{(20)}=-1.259, p=.222\right)$. The ANOVAs yielded a significant main effect of relatedness in the midline within the N400 time window $\left(F(1,20)=7.892, p=.032, \eta_{p}{ }^{2}=.209\right)$ and a trend within the LPC time window $\left(F(1,20)=3.719, p=.068, \eta_{p}{ }^{2}=.157\right)$, while none of the interactions between relatedness and hemisphere/anteriority at the midline or lateral clusters was significant $(p s>.10)$.

\subsection{Perturbation of old knowledge}

The perturbation effect was examined on probes that were related to the original meaning of the preceding known word when that word had been associated with a new meaning, compared with when it merely had been exposed (e.g., plenty - enough vs. weapon - war). As shown in Fig. 4B, probes related to known words with new meanings evoked a larger negativity within the LPC time window $\left(t_{(20)}=-2.555, p=.019\right)$. The difference within N400 time window had the same direction but did not reach statistical significance $\left(t_{(20)}=-1.354, p=.167\right)$. A main effect of condition in the midline that was marginally significant within N400 time window $\left(F(1,20)=3.055, p=.096, \eta_{p}{ }^{2}=.133\right)$ reached significance within the later window $(F(1,20)=6.845, p=.017$, $\left.\eta_{p}{ }^{2}=.255\right)$. Additionally, there was a significant interaction between condition and hemisphere at the lateral clusters driven by a left lateralized negativity within $\mathrm{N} 400$ time window $(F(2,40)=3.688$, $\left.p=.034, \eta_{p}{ }^{2}=.216\right)$. The interaction had the same pattern within the later time window although it was not significant $(F(1,20)=2.828$, $\left.p=.109, \eta_{p}{ }^{2}=.124\right)$. To explore whether the observed perturbation effect is related to performance in the meaning generation or multiplechoice test, we calculated the Pearson correction between the magnitude of perturbation effect (the difference between the two conditions in mean amplitudes within 500-700 ms) and performance on known words on Day 1, Day 8, and retention ratio in each test. However, none was significant (all $p s>.48$ in both tasks).

\subsection{Integration of new knowledge}

To examine whether the newly learned meanings were able to prime semantically related words, we compared probes that were related to the trained meaning of a novel word with probes that were unrelated (e.g., tasdite - busy vs. tasdite - paper). For real words, we compared probes related to their new meanings with probes unrelated to the meanings of exposure controls (e.g., plenty - germ vs. weapon - door). As shown in Fig. 5, new-meaning probes of novel words did not show a reduced negativity within the $\mathrm{N} 400\left(300-500 \mathrm{~ms}: t_{(20)}=-.998\right.$, $p=.330)$ or the LPC time window $\left(t_{(20)}=-.800, p=.433\right)$. In the ANOVAs, there was a marginally significant interaction between relatedness and anteriority for novel words in the midline within the N400 time window $\left(F(2,40)=2.540, p=.080, \eta_{p}{ }^{2}=.119\right)$, reflecting a posterior positivity for the unrelated probes compared to related probes. For known words, there was also no difference between related and unrelated probes in either time window ( $p s>.61$ ). The main effect of relatedness and the interaction between relatedness and anteriority/ hemisphere were not significant in either time window ( $p s>.45)$. 


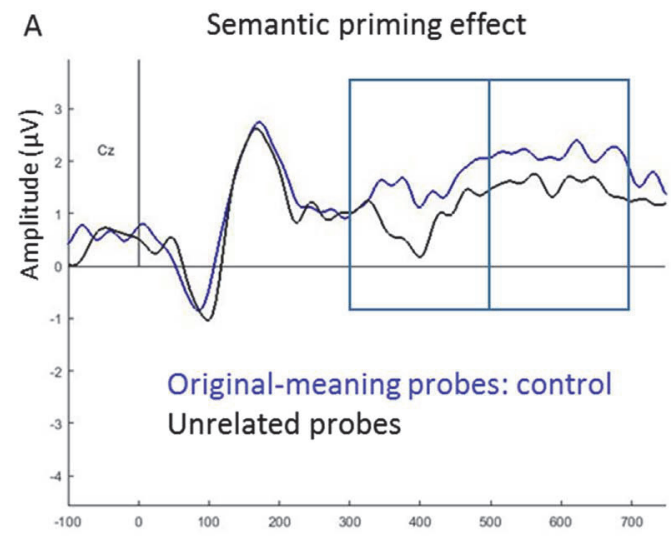

unrelated - related

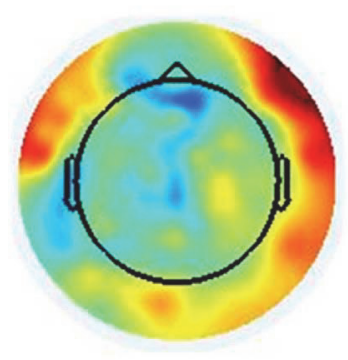

$300-500 \mathrm{~ms}$ unrelated - related

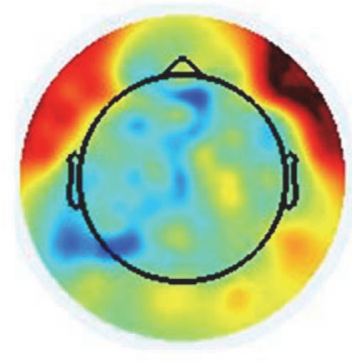

$500-700 \mathrm{~ms}$
B

Perturbation effect

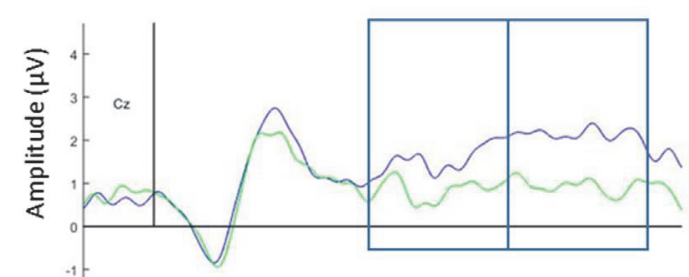

Original-meaning probes: control

Original-meaning probes: meaning

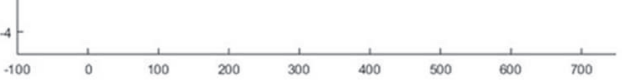

meaning - control

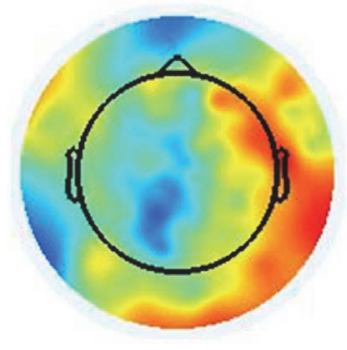

$300-500 \mathrm{~ms}$

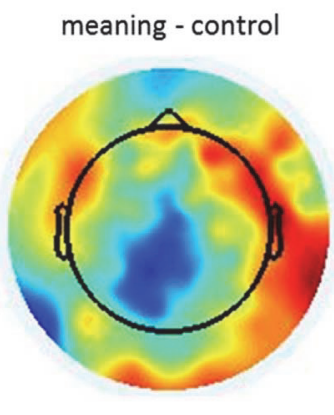

$500-700 \mathrm{~ms}$

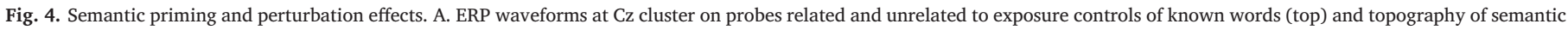

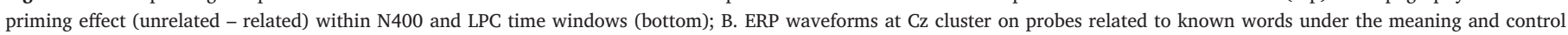
conditions (top) and topography of perturbation effect (meaning - control) within N400 and LPC time windows (bottom).

A

Integration on novel words

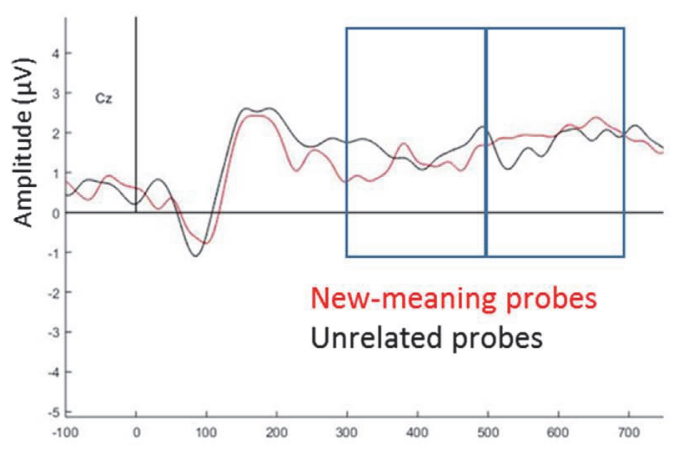

Unrelated - related

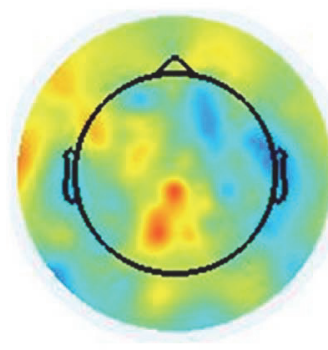

$300-500 \mathrm{~ms}$ unrelated - related

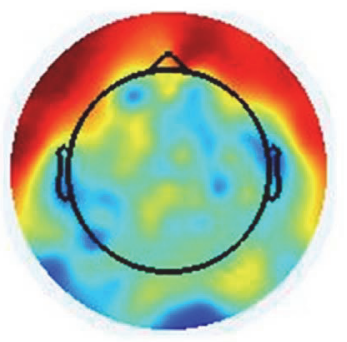

$500-700 \mathrm{~ms}$

B

Integration on known words

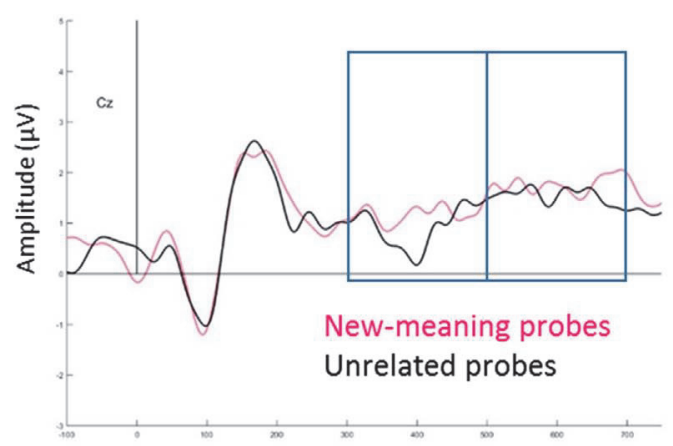

unrelated - related

unrelated - related

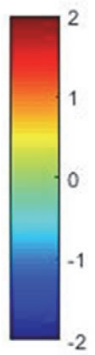

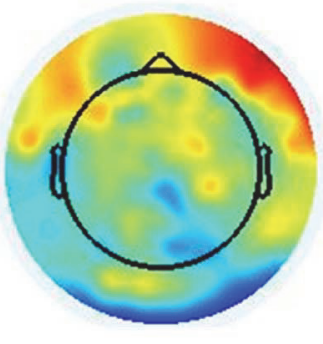

$300-500 \mathrm{~ms}$

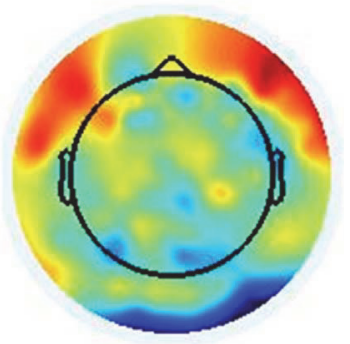

500-700 ms

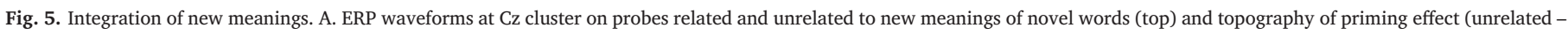

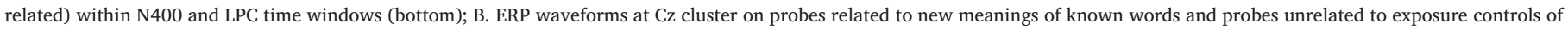
known words (top) and topography of relatedness effect (unrelated - related) within N400 and LPC time windows (bottom). 


\section{Discussion}

Participants learned new meanings for known words better than novel words, and maintained the memories of new meanings of known words better over one week, as measured in the meaning generation tests. Complementing this general behavioral picture are ERPs recorded on trained words including exposure controls and on following probe words that were related to new trained meanings, original meanings, or unrelated during a one-back task. The ERP results provide windows on the on-line processing of these words when participants did not need to process their meanings, thus capturing strictly implicit meaning processes that should be evident when meanings have been added to semantic memory (consolidated). The N400 and LPC can indicate lexico-semantic processing, with more negative N400 or reduced late positivity reflecting more effortful meaning access and less meaning congruence.

Our data showed no N400 reduction on new-meaning probes of known or novel words (Fig. 5), indicating the absence of semantic priming from new meanings or meaning integration on the day of learning. In contrast, original-meaning probes showed a larger negativity especially within the LPC time window when the preceding known words had been paired with new meanings, compared to when they had been presented as exposure controls (Fig. 4B), indicating reduced semantic priming from the original meanings. This result suggests that the original meaning of a trained word was less accessible following attempts to learn a new meaning. ERPs on trained words also argue for reduced accessibility of original meanings - larger negativity within both N400 and LPC time windows for known words with new meanings than their exposure controls. In contrast, no such pattern occurred for novel words, which lack original meanings (Fig. 3). The differences indicate that meaning access of known words had been affected by learning new meanings.

One important feature of integration is that new knowledge can interact with existing knowledge directly (Davis and Gaskell, 2009). In the current case, the new meaning of a word can effectively prime semantically related words. Consistent with a previous study using a primed lexical decision task (Tamminen and Gaskell, 2013), we did not find a semantic priming effect for novel words and their new-meaning probes in the one-back task (Fig. 5A), although the paradigm is able to detect semantic relatedness among existing words (Fig. 4A). This cannot be attributed to the lack of knowledge about the new meanings, as behavioral data indicate that participants were able to recall and recognize the new meanings. Additionally, the new meanings and probes were rated as highly related (see section 2.2.2) and participants were able to make semantic relatedness judgments on the word pairs, as found in a previous study (Fang et al., 2016). Instead, the absence of priming indicates that prior to overnight consolidation, the connections of novel words with their meanings are essentially memories of the learning episodes, rather than "semantic" memories (Davis and Gaskell, 2009). Although participants had better performance for known words than novel words in both behavioral tests, we also found no evidence of priming for the new meanings of known words on the day of learning (Fig. 5B). This finding indicates that consolidation is also necessary for new meanings to become part of semantic memory, at least when they are unrelated to original meanings,

Previous studies using a semantic relatedness judgment task reported a reduced negativity within N400 and/or LPC time window on new-meaning probes on the day of learning (Bakker et al., 2015; Mestres-Misse et al., 2007; Perfetti et al., 2005). Our contrasting result comes from a paradigm that minimized explicit meaning processing, raising the possibility that semantic priming effects in the previous studies could be attributed to their requirement for explicit meaning retrieval. In addition to this task difference, experimental stimuli may also contribute to the absence of priming effect in the current study. In the two studies reporting N400 effect, new-meaning probes were either real-word counterparts (i.e., hidden words) of novel words (Mestres-
Misse et al., 2007) or part of definitions (Perfetti et al., 2005), thus making the meaning relations between trained words and probes more explicit and allowing probes to be activated as part of learning episodes. Similar to the current study, probes in both Bakker et al. (2015) and Tamminen and Gaskell (2013) were not semantic equivalents of new meanings and never occurred in the definitions. Bakker et al. (2015) found semantic priming within the LPC but not the N400 time window, whereas Tamminen and Gaskell (2013) and the current study used tasks that did not rely on explicit semantic processing of the stimuli and did not observe any robust priming effect.

Although ERP indicators suggest that integration of the new meanings did not occur, they do suggest an effect of learning new meanings on access to the original meanings. The larger negativity on originalmeaning probes of known words with new meanings (relative to those of exposure controls, Fig. 4B) reflects a perturbation of existing wordmeaning connections. Especially interesting is that this perturbation effect emerges despite the fact that the prior word knowledge is wellestablished and that the task requires no access to meaning. Thus, what emerged is best characterized an automatic perturbation of the existing form-meaning connection, rather than a strategic process.

Evidence for perturbation comes also from ERPs recorded on the trained words. The larger negativity for known words with new meanings relative to their exposure controls at the central midline within both N400 and LPC time windows (Fig. 3) may reflect reduced accessibility of original meanings or more effortful meaning access. The larger negativity is similar to what was observed in less accessible low frequency words in contrast to high frequency words (Rugg, 1990). However, we cannot completely exclude the possibility that new meanings are activated at the presence of words. In that case, the larger negativity on known words with new meanings may reflect the influence of the richer information that is available after meaning learning (Lee and Federmeier, 2006, 2008). Alternatively, it may reflect competition between meanings, as occurs for semantically ambiguous words (Kellenbach et al., 2002). However, given the absence of semantic priming from the new meanings, the activation of new meanings for trained words is very weak, and perhaps not comparable to the multiple meanings of an ambiguous word.

To place the perturbation interpretation in the context of previous research on word learning and consolidation, we note that most previous research has demonstrated consolidation by manipulating form or meaning relations between new with existing knowledge (e.g., Bakker et al., 2014; Bowers et al., 2005; Coutanche and Thompson-Schill, 2014; Dumay and Gaskell, 2007; Qiao and Forster, 2013; Tamminen and Gaskell, 2013; Tham et al., 2015). These studies examine lexical competition between novel words and existing words such as "cathedruke" and "cathedral" or semantic priming between novel words and existing words such as "tasdite" and "busy" here. Consolidation is important to build up stable connections between new and existing knowledge (Davis and Gaskell, 2009). However, we suggest the perturbation of existing connections does not necessarily coincide with the integration of new knowledge. In conditions represented by the current study-one form, two unrelated meanings - this perturbation of meaning 1 occurs before integration of meaning 2 .

An interesting question is the function, if any, of form-meaning perturbation. Memories, including those for original word meanings, can become labile when they are reactivated, and this reactivation makes the incorporation of new information possible (Forcato et al., 2010). The reduced accessibility of established connections with the original meanings might be a necessary early phase of integrating new connections with an existing word form. A related idea is that the perturbation results from the suppression of original meanings during the learning phase to allow new associations to be encoded. Both possibilities argue for the functionality of the perturbation effect. However, in our sample we did not see a significant correlation between magnitude of the perturbation effect within LPC time window and any of the learning measures of known words in our exploratory 
analyses. We suggest that it is the effort at learning a new meaning, rather than the result of the learning, that leads to the perturbation of an original meaning. If so, the integration of new knowledge may be relatively independent of any perturbation of existing knowledge, which would then merely be an early by-product of trying to associate new unrelated meanings to known words. Further research is required to examine whether the perturbation of an existing lexico-semantic network directly contributes to the integration of new knowledge. Also unclear is whether perturbation occurs when new meanings are related to the original meanings. New related meanings may be integrated faster than unrelated meanings, as studied behaviorally in Rodd et al. (2012). It is possible that the learning process for related meanings proceeds with integration-only, without disturbance of the original meaning.

Finally, learning a novel word and learning new meaning for a known word may involve different learning mechanisms. van Kesteren et al. (2012) proposed that neocortex-based learning is more functional than hippocampus-based learning when new information is congruent with prior knowledge. In the current study, the new meanings of known words are "incongruent" with existing knowledge. However, the activation of prior word knowledge in neocortex along with encoding the new meaning may lead to stronger interaction between hippocampus and neocortex, facilitating the integration of new meanings and their retention. Although we did not see immediate integration of new meanings for known words, we did see a better long-term retention and an immediate perturbation effect as a result of learning new meanings. In the case of novel words, less existing knowledge is activated, leaving the hippocampus to play a dominant role. This explanation is also consistent with our findings for the $\mathrm{N} 400$ on trained words. At $\mathrm{Cz}$, novel words with new meanings did not differ from exposure controls and both showed a more negative N400 than merely exposed real words. Other research shows that mere exposure to novel words does not eliminate the more negative N400 they show, relative to real words (Bermúdez-Margaretto et al., 2014). Our study suggests that even with meaning training, novel words and real words continue to show this N400 difference on the day of learning.

In conclusion, the results of our study expose an immediate influence of learning new meanings on existing word knowledge. The one-back task, which does not require explicit meaning retrieval, revealed ERP effects that require an interpretation of reduced accessibility of the original meaning following attempts to learn a new meaning. Whereas learning novel words is a process of adding new form-meaning pairs to the vocabulary, learning a new meaning for an existing word brings about a dynamic perturbation of the existing meaning connections. This effect can occur relatively early in learning before the new knowledge is integrated into the existing network.

\section{Acknowledgement}

This research was supported by National Science Foundation Grant SBE08-36012 through the Pittsburgh Science of Learning Center and by NIH award 1R01HD058566-01A1 to the University of Pittsburgh (C. Perfetti, PI). The authors would like to thank Christine Shi for help with stimuli development and data collection, Hannah Legerwood, Austin Marcus and Kimberly Muth for help with data collection, and the two anonymous reviewers for constructive comments. Part of this work was presented at the Seventh Annual Meeting of the Society for Neurobiology of Language, Chicago, IL, 2015.

\section{References}

Bakker, I., Takashima, A., van Hell, J.G., Janzen, G., McQueen, J.M., 2014. Competition from unseen or unheard novel words: lexical consolidation across modalities. J. Mem. Lang. 73 (0), 116-130. http://dx.doi.org/10.1016/j.jml.2014.03.002.

Bakker, I., Takashima, A., van Hell, J.G., Janzen, G., McQueen, J.M., 2015. Tracking lexical consolidation with erps: lexical and semantic-priming effects on $\mathrm{n} 400$ and lpc responses to newly-learned words. Neuropsychologia 79 (Pt A), 33-41. http://dx.doi. org/10.1016/j.neuropsychologia.2015.10.020.

Batterink, L., Neville, H., 2011. Implicit and explicit mechanisms of word learning in a narrative context: an event-related potential study. J. Cogn. Neurosci. 23 (11), 3181-3196. http://dx.doi.org/10.1162/jocn a 00013.

Bermúdez-Margaretto, B., Beltrán, D., Domínguez, A., Cuetos, F., 2014. Repeated exposure to "meaningless" pseudowords modulates lpc, but not n (fn) 400. Brain Topogr. 1-14.

Bowers, J.S., Davis, C.J., Hanley, D.A., 2005. Interfering neighbours: the impact of novel word learning on the identification of visually similar words. Cognition 97 (3), B45-B54. http://dx.doi.org/10.1016/j.cognition.2005.02.002.

Brysbaert, M., New, B., 2009. Moving beyond kucera and francis: a critical evaluation of current word frequency norms and the introduction of a new and improved word frequency measure for american english. Behav. Res. Methods 41 (4), 977-990. http://dx.doi.org/10.3758/BRM.41.4.977.

Brysbaert, M., Warriner, A.B., Kuperman, V., 2013. Concreteness ratings for 40 thousand generally known english word lemmas. Behav. Res. Methods 1-8.

Cavanagh, J., Geisler, M.W., 2006. Mood effects on the erp processing of emotional intensity in faces: a p3 investigation with depressed students. Int. J. Psychophysiol. 60 (1), 27-33. http://dx.doi.org/10.1016/j.ijpsycho.2005.04.005.

Chwilla, D.J., Kolk, H.H.J., 2003. Event-related potential and reaction time evidence for inhibition between alternative meanings of ambiguous words. Brain Lang. 86 (2), 167-192.

Coutanche, M.N., Thompson-Schill, S.L., 2014. Fast mapping rapidly integrates information into existing memory networks. J. Exp. Psychol.-Gen. 143 (6), 2296-2303. http://dx.doi.org/10.1037/xge0000020.

Davis, M.H., Gaskell, M.G., 2009. A complementary systems account of word learning: neural and behavioural evidence. Philos. Trans. R. Soc. Lond. B Biol. Sci. 364 (1536), 3773-3800. http://dx.doi.org/10.1098/rstb.2009.0111.

Dien, J., 2010. The ERP PCA toolkit: an open source program for advanced statistical analysis of event-related potential data. J. Neurosci. Methods 187 (1), 138-145. http://dx.doi.org/10.1016/j.jneumeth.2009.12.009.

Dumay, N., Gaskell, M.G., 2007. Sleep-associated changes in the mental representation of spoken words. Psychol. Sci. 18 (1), 35-39. http://dx.doi.org/10.1111/j.1467-9280. 2007.01845.x.

Fang, X., Perfetti, C., Stafura, J., 2016. Learning new meanings for known words: biphasic effects of prior knowledge. Lang. Cogn. Neurosci.

Forcato, C., Rodriguez, M.L., Pedreira, M.E., Maldonado, H., 2010. Reconsolidation in humans opens up declarative memory to the entrance of new information. Neurobiol. Learn. Mem. 93 (1), 77-84. http://dx.doi.org/10.1016/j.nlm.2009.08.006.

Forcato, C., Burgos, V.L., Argibay, P.F., Molina, V.A., Pedreira, M.E., Maldonado, H., 2007. Reconsolidation of declarative memory in humans. Learn. Mem. 14 (4), 295-303. http://dx.doi.org/10.1101/lm.486107.

Geukes, S., Gaskell, M.G., Zwitserlood, P., 2015. Stroop effects from newly learned color words: effects of memory consolidation and episodic context. Front. Psychol. 6, 278. http://dx.doi.org/10.3389/fpsyg.2015.00278.

Hill, H., Ott, F., Weisbrod, M., 2005. SOA-dependent N400 and P300 semantic priming effects using pseudoword primes and a delayed lexical decision. Int. J. Psychophysiol. 56 (3), 209-221.

Hill, H., Strube, M., Roesch-Ely, D., Weisbrod, M., 2002. Automatic vs. controlled processes in semantic priming - differentiation by event-related potentials. Int. J. Psychophysiol. 44 (3), 197-218.

Kellenbach, M.L., Wijers, A.A., Hovius, M., Mulder, J., Mulder, G., 2002. Neural differentiation of lexico-syntactic categories or semantic features? Event-related potential evidence for both. J. Cogn. Neurosci. 14 (4), 561-577. http://dx.doi.org/ 10.1162/08989290260045819.

Koen, J.D., Rugg, M.D., 2016. Memory reactivation predicts resistance to retroactive interference: evidence from multivariate classification and pattern similarity analyses. J. Neurosci. 36 (15), 4389-4399. http://dx.doi.org/10.1523/jneurosci. 4099-15.2016.

Kutas, M., Federmeier, K.D., 2011. Thirty years and counting: finding meaning in the n400 component of the event-related brain potential (erp). Annu. Rev. Psychol. 62, 621-647. http://dx.doi.org/10.1146/annurev.psych.093008.131123.

Landauer, T.K., Dumais, S.T., 1997. A solution to plato's problem: the latent semantic analysis theory of acquisition, induction, and representation of knowledge. Psychol. Rev. 104 (2), 211-240. http://dx.doi.org/10.1037//0033-295x.104.2.211.

Lee, C.L., Federmeier, K.D., 2006. To mind the mind: an event-related potential study of word class and semantic ambiguity. Brain Res. 1081 (1), 191-202. http://dx.doi.org/ 10.1016/j.brainres.2006.01.058

Lee, C.L., Federmeier, K.D., 2008. To watch, to see, and to differ: an event-related potential study of concreteness effects as a function of word class and lexical ambiguity. Brain Lang. 104 (2), 145-158.

Lindsay, S., Gaskell, M.G., 2013. Lexical integration of novel words without sleep. J. Exp. Psychol. Learn. Mem. Cogn. 39 (2), 608-622. http://dx.doi.org/10.1037/a0029243.

Mcclelland, J.L., Mcnaughton, B.L., Oreilly, R.C., 1995. Why there are complementary learning-systems in the hippocampus and neocortex - insights from the successes and failures of connectionist models of learning and memory. Psychol. Rev. 102 (3), 419-457. http://dx.doi.org/10.1037/0033-295x.102.3.419.

McNamara, T.P., 2005. Semantic Priming: Perspectives From Memory and Word Recognition. Psychology Press.

Mestres-Misse, A., Rodriguez-Fornells, A., Munte, T.F., 2007. Watching the brain during meaning acquisition. Cereb. Cortex 17 (8), 1858-1866 (doi: bhl094) (pii) (10.1093/ cercor/bhl094).

Parks, R., Ray, J., Bland, S., 1998. Wordsmyth English Dictionary - Thesaurus. University of Chicago.

Perfetti, C.A., Wlotko, E.W., Hart, L.A., 2005. Word learning and individual differences in word learning reflected in event-related potentials. J. Exp. Psychol. Learn. Mem. 
Cogn. 31 (6), 1281-1292. http://dx.doi.org/10.1037/0278-7393.31.6.1281.

Qiao, X., Forster, K.I., 2013. Novel word lexicalization and the prime lexicality effect. J. Exp. Psychol. Learn. Mem. Cogn. 39 (4), 1064-1074. http://dx.doi.org/10.1037/ a0030528.

Rodd, J.M., Berriman, R., Landau, M., Lee, T., Ho, C., Gaskell, M.G., Davis, M.H., 2012. Learning new meanings for old words: effects of semantic relatedness. Mem. Cogn. 40 (7), 1095-1108. http://dx.doi.org/10.3758/s13421-012-0209-1.

Rugg, M.D., 1990. Event-related brain potentials dissociate repetition effects of high- and low-frequency words. Mem. Cogn. 18 (4), 367-379.

Schlichting, M.L., Preston, A.R., 2015. Memory integration: neural mechanisms and implications for behavior. Curr. Opin. Behav. Sci. 1, 1-8. http://dx.doi.org/10.1016/ j.cobeha.2014.07.005.

Schlichting, M.L., Mumford, J.A., Preston, A.R., 2015. Learning-related representational changes reveal dissociable integration and separation signatures in the hippocampus and prefrontal cortex. Nat. Commun. 6, 8151. http://dx.doi.org/10.1038/ ncomms9151.

Tamminen, J., Gaskell, M.G., 2013. Novel word integration in the mental lexicon: evidence from unmasked and masked semantic priming. Q. J. Exp. Psychol. 66 (5), 1001-1025. http://dx.doi.org/10.1080/17470218.2012.724694.

Tham, E.K., Lindsay, S., Gaskell, M.G., 2015. Markers of automaticity in sleep-associated consolidation of novel words. Neuropsychologia. http://dx.doi.org/10.1016/j. neuropsychologia.2015.03.025.

van Kesteren, M.T.R., Ruiter, D.J., Fernandez, G., Henson, R.N., 2012. How schema and novelty augment memory formation. Trends Neurosci. 35 (4), 211-219. http://dx. doi.org/10.1016/j.tins.2012.02.001.

Zachau, S., Korpilahti, P., Hamalainen, J.A., Ervast, L., Heinanen, K., Suominen, K., Lehtihalmes, M., Leppänen, P.H., 2014. Electrophysiological correlates of crosslinguistic semantic integration in hearing signers: N400 and lpc. Neuropsychologia 59, 57-73. 